\title{
Josephson tunneling between weakly interacting Bose-Einstein condensates
}

\author{
F. Meier and W. Zwerger \\ Sektion Physik, Universität München, Theresienstrasse 37, D-80333 München, Germany
}

(October 15, 2018)

\begin{abstract}
Based on a tunneling Hamiltonian description, we calculate the Josephson, normal and interference currents between two Bose-Einstein condensates described by the Bogoliubov theory. The dominant Josephson term is of first order in the tunneling with a critical current density proportional to the ground state pressure. In contrast to superconductors, the normal current remains finite at zero temperature. We discuss the dynamics of the relative phase in a semiclassical approximation derived from an exact functional integral approach, which includes the interaction effects at fixed total particle number. It is shown that the normal current leads to a damping of the Josephson oscillations and, at long times, eliminates the macroscopic quantum self trapping predicted by Smerzi et.al. Finally we give estimates for an experimental realization of Josephson tunneling in cold atomic gases, which indicate that coherent transfer of atoms might be realized with a ${ }^{23} \mathrm{Na}$ condensate.
\end{abstract}

03.75.Fi,05.30.Jp,74.50.+r

\section{INTRODUCTION}

The realization of Bose-Einstein condensation (BEC) in atomic vapors [1] provides an example of superfluidity, to which the approximation of a weakly interacting Bose gas applies quantitatively. Due to the low densities $n \approx 10^{14} \mathrm{~cm}^{-3}$ and with typical scattering lengths $a \approx 5 \mathrm{~nm}$, the gas parameter $n a^{3}$ is of order $10^{-5}$, i.e. well in the range of applicability of the Bogoliubov theory. Although the interactions still entail strong quantitative changes compared to an ideal gas picture [ [ are rather well described by the weak coupling GrossPitaevski equation (GPE) and its small fluctuations, the Bogoliubov equations [5]. Many of the phenomena predicted within this framework like sound modes with a linear spectrum have been established experimentally [6]. More recently genuine superfluid properties like quantized vortices $[7,8]$ or the existence of a critical velocity [9] have also been observed.

The Josephson effect 10,11 is one of the prime examples of macroscopic quantum effects, displaying directly the broken symmetry associated with the relative phase of two weakly coupled condensates [12]. In superconductors it is a rather standard phenomenon, in contrast to Bose condensed systems, where it has first been observed by Avenel and Varoquaux in superfluid ${ }^{4} \mathrm{He}[13$. For dilute atomic gases, coherent oscillations have been seen in driven two component BECs 14 and in vertical arrays of traps of an optical lattice 15. However these are Rabi- or Wannier-Stark-type oscillations and should not be confused with a genuine ac-Josephson effect as discussed here. Theoretically, the Josephson effect in this context has been investigated by Smerzi et al. [16, who focussed on the nonlinear dynamics of the relative phase and population difference. Zapata et al. [17] calculated the Josephson coupling energy due to condensatecondensate tunneling for weakly coupled harmonic wells and gave estimates for the normal currents due to the thermal cloud. These studies were essentially limited to a mean field like Gross-Pitaevski description, neglecting noncondensate contributions. More recently Villain and Lewenstein [18] showed that particles out of the condensate lead to a damping of the relative phase in a two component condensate with a Josephson like coupling induced by the external driving field [14].

Our aim in this work is to provide a microscopic calculation of the Josephson effect between two weakly interacting BECs separated by a tunnel barrier 19. Using an idealized model, we give quantitative results for both the superfluid and normal currents per area which only depend on the microscopic parameters of the condensate and the barrier transmission amplitude. Moreover, a complete theory is given for the interaction effects arising in coupled condensates with a fixed total particle number in the limit, where the number of exchanged particles is small. The associated dynamics is discussed within a semiclassical approximation. Using realistic parameters in dilute atomic gases, the effects due to a fixed number of particles are appreciable, but do not destroy the qualitative structure of oscillating Josephson currents for condensates at different chemical potentials. Our estimates indicate that the Josephson effect might be observable with currently available techniques for ${ }^{23} \mathrm{Na}$ or lighter atoms.

The outline of the paper is as follows: In section 2, we introduce the tunneling Hamiltonian model, including a discussion of the relevant states in condensates with a fixed total particle number and the general form of the Josephson coupling energy. This energy and the associated nondissipative current is calculated up to second order in the tunneling matrix elements in section 3. For a finite, given difference in the chemical potentials we determine in section 4 the complete particle current in terms of the normal and anomalous spectral functions of the individual condensates. In section 5 , we develop an exact functional integral representation of the reduced 
quantum mechanics of weakly coupled Bose condensates which incorporates the effect of interactions at fixed total particle number. In section 6 , explicit results are given for an idealized geometry, determining the physically relevant currents per area from microscopic parameters which characterize the separate condensates. The resulting semiclassical dynamics of the relative phase, including 'charging' effects and dissipation, is solved numerically in section 7 for parameters, which are realistic for current condensates of dilute atomic gases. Moreover, we test the tunneling Hamiltonian Ansatz by comparing it with exact numerical results for the one-dimensional GPE with a barrier. Conclusions and open questions are discussed in section 8 .

\section{THE TUNNELING HAMILTONIAN}

Conceptually, the most obvious way to realize a Josephson geometry for BECs with current experimental setups, is to split a single condensate in a long trap into two separate parts by a narrow light sheet produced by a blue detuned laser. The repulsive potential due to the ac-Stark shift is proportional to the laser intensity and thus the height and width of the barrier may be varied in a considerable range. In the limit of a strong barrier one has to zeroth order two completely separate condensates $a$ and $b$ with Hamiltonians $\hat{H}_{a / b}$. Provided that the coupling energy $E_{J}$ due to the transfer of particles across the barrier is small compared to the ground state energies of $\hat{H}_{a / b}$, which are of order $\mu N$, the coupled system may then be approximated by a tunneling or transfer Hamiltonian [20]

$$
\hat{H}=\hat{H}_{a}+\hat{H}_{b}+\hat{H}_{T}
$$

in which the contribution $\hat{H}_{T}=\hat{\Lambda}+\hat{\Lambda}^{\dagger}$ describing the transfer of particles between $a$ and $b$ can be treated perturbatively. Formally, introducing a set of orthonormal eigenstates $\{|l\rangle\}$ and $\{|r\rangle\}$ which decay exponentially in the barrier where $a$ and $b$ overlap, an instantaneous transfer is described by a term

$$
\hat{\Lambda}=-\sum_{l, r} t_{l r} \hat{a}_{l}^{\dagger} \hat{a}_{r}
$$

with tunneling amplitudes $t_{l r}$, which may be expressed in terms of current matrix elements between the left and right eigenstates 20] (see section 6 below). The signs here have been choosen in such a way that for positive, real ground state wave functions in $a$ and $b$, the associated tunneling amplitude is positive, giving rise to a lowering of the ground state energy in the coupled system, as expected in a standard double well situation. While a rigorous derivation of the transfer Hamiltonian is rather difficult 20], it is expected to be valid for high and narrow barriers, for which the mean field interaction of the condensate is negligible within the barrier. This is in fact confirmed through numerical calculations in an exactly soluble one-dimensional geometry in section 7 . Note that the states $\{|l\rangle\}$ and $\{|r\rangle\}$ introduced in Eq. (2) are not mutually orthogonal, and thus $\hat{H}_{a}$ and $\hat{H}_{b}$ do not commute beyond zeroth order in $\hat{H}_{T}$ [21]. Moreover, the creation and annihilation operators in (2) refer to the transfer of atoms, not that of quasiparticles, which are the proper excitations only within the individual condensates.

As pointed out by Ferrell and Prange [22], the essence of the Josephson effect is that in the absence of any drop $\Delta \mu$ in the chemical potential between both sides, no energy is required to transfer a condensate atom across the barrier (note that a finite value of $\Delta \mu$ may be present either externally e.g. by a drop in the gravitational potential as in the experiment by Anderson and Kasevich [15], or may arise internally at finite transfer currents through 'charging' effects discussed in section 5 below). Thus the states $|\nu\rangle=\left|\bar{N}_{a}+\nu, \bar{N}_{b}-\nu\right\rangle$ in which an arbitrary integer number $\nu$ of condensate atoms have tunneled from right to left, are degenerate. The ground state of the coupled system is therefore not an eigenstate $|\nu\rangle$ with a definite relative particle number, but rather a coherent superposition

$$
|\varphi\rangle=\sum_{\nu} c_{\nu} e^{i \nu \varphi}|\nu\rangle
$$

with a definite relative phase $\varphi=\varphi_{a}-\varphi_{b}$. Here the $c_{\nu}$ are real coefficients obeying $\sum c_{\nu}^{2}=1$ which are constant around $\nu=0$, decaying to zero only at $|\nu|$ of order $\sqrt{N_{a / b}}$. In terms of the eigenstates $\left|\varphi_{a}, \varphi_{b}\right\rangle$ in which each of the condensates has a definite overall phase $\varphi_{a, b}$, the state $|\varphi\rangle$ can be expressed in the form

$$
|\varphi\rangle \propto \int_{0}^{2 \pi} \frac{d \varphi_{b}}{2 \pi} e^{-i\left(N_{a}+N_{b}\right) \varphi_{b}}\left|\varphi_{b}+\varphi, \varphi_{b}\right\rangle,
$$

using the standard relation between states with a fixed phase or a fixed particle number [12]. The state with a definite relative phase is thus a projection of states $\left|\varphi_{a}, \varphi_{b}\right\rangle$ with a broken gauge symmetry in the individual condensates to one with a definite total particle number $N_{a}+N_{b}$.

The delocalization in the relative particle number $\nu$ described by the coherent state $|\varphi\rangle$, gives rise to a lowering of the energy of the coupled condensates in the same manner, in which a Bloch state gains kinetic energy by spreading over many sites in a periodic potential with localized Wannier orbitals labeled by $\nu$. In a time reversal invariant situation, this energy (or free energy at $T \neq 0$ ) must be even and periodic in $\varphi \rightarrow \varphi+2 \pi$. Quite generally it can thus be expanded in a Fourier series of the form 23]

$$
E(\varphi)=\sum_{n=0}^{\infty} E_{n} \cos n \varphi .
$$

Since $\nu$ and $\hbar \varphi$ are canonically conjugate variables, the nondissipative current 


$$
I(\varphi)=-\frac{d \nu}{d t}=-\frac{\partial E(\varphi)}{\partial \hbar \varphi}=\sum_{n=1}^{\infty} I_{n} \sin n \varphi
$$

can be obtained from the knowledge of the phase dependent part of the coupling energy between the two condensates. Within a tunneling Hamiltonian description, the coefficients $E_{n}=\hbar I_{n} / n$ are of order $\left|\hat{H}_{T}\right|^{n}$. It is thus usually sufficient to keep only the lowest nonvanishing term, conventionally denoted by $-E_{J}$, which is negative in the standard situation, where the lowest energy state is that with a vanishing relative phase $\varphi=0$. As will be shown below, for Bose condensates the Josephson coupling arises already in first order in the tunneling amplitudes,

$$
<\varphi\left|\hat{H}_{T}\right| \varphi>=-E_{J} \cos \varphi
$$

with a positive Josephson coupling energy $E_{J}$, which immediately determines the associated critical current $I_{c}=E_{J} / \hbar$. For superconductors, in turn, where the Josephson effect is associated with the coherent tunneling of pairs, the first order term vanishes and $E_{J}$ is proportional to the average of $\left|t_{l r}\right|^{2}$ at the Fermi energy. The latter being a direct measure also of the normal currents, one obtains the well known relation

$$
2 E_{J}^{B C S}=\Delta \tanh \beta \Delta / 2 \cdot h G_{n} / 4 e^{2}
$$

connecting the BCS Josephson coupling to the superconducting gap $\Delta$ and the dimensionless normal state conductance $G_{n}$ [24].

Since tunneling is a small perturbation, the fact that there is a Josephson effect already in first order for a Bose condensed system, might seem to make higher order calculations unnecessary in this case. In fact, there are two reasons why this is not so: First of all, it turns out that realistic barriers which allow to observe a Josephson effect in coupled BECs have to be small enough, that higher order effects are nonnegligible. More importantly, though, it is only at second order that dissipative effects appear through normal currents which, as we will see, can have strong effects even if they are small.

\section{THE PHASE DEPENDENT COUPLING ENERGY}

In order to calculate the change in energy due to tunneling, we employ perturbation theory up to second order in the transfer term $\hat{H}_{T}$. To be specific, we assume that the condensate size is large compared with the coherence length $\xi=(8 \pi n a)^{-1 / 2}$ over which the condensate wave function varies [4]. Under this condition, which is well realized in present gaseous BECs, the eigenstates $|l\rangle$ and $|r\rangle$ introduced above for a weakly interacting gas are given by

- the condensate ground states $\phi_{a / b}(x / y)$ which follow from a solution of the Gross-Pitaevski equation in the given confining potential, and
- a set of excited states orthogonal to $\phi_{a / b}(x / y)$, which may be labeled by nonzero wavenumbers $k$ or $q$ for condensates $a$ and $b$ respectively. These states are obtained by solving the standard Bogoliubovequations in a local density or - equivalently - a semiclassical approximation, resulting in wavefunctions and energies like that in a homogeneous system with a local condensate density $n_{c}(x)$ 25].

The transfer operator

$$
\begin{aligned}
\hat{\Lambda}=-t_{c c} \hat{a}_{c, a}^{\dagger} \hat{a}_{c, b} & -\sum_{k} t_{k c} \hat{a}_{k}^{\dagger} \hat{a}_{c, b} \\
& -\sum_{q} t_{c q} \hat{a}_{c, a}^{\dagger} \hat{a}_{q}-\sum_{k, q} t_{k q} \hat{a}_{k}^{\dagger} \hat{a}_{q}
\end{aligned}
$$

is thus split into contributions describing condensate to condensate (c-c), condensate to noncondensate (cnc) and noncondensate to noncondensate (nc-nc) tunneling. To first order in $\hat{H}_{T}$, only the c-c term contributes and gives rise to an energy gain of precisely the form (7), with a positive Josephson coupling energy $E_{J}^{B E C}=2 t_{c c}\left(\bar{N}_{a} \bar{N}_{b}\right)^{1 / 2}$, which favors a fixed relative phase $\varphi=0$ in the ground state of the coupled system. Here, as in the rest of our work, we have assumed that $|\nu|$ remains much smaller than the average number $\bar{N}_{a / b}$ of condensate atoms in each well. The Josephson coupling energy is thus independent of the number of transferred atoms to lowest order.

For the calculation of the second order energy shift

$$
\Delta E^{(2)}=-\sum_{e} \frac{\left|\left\langle e\left|\hat{H}_{T}\right| 0\right\rangle\right|^{2}}{E_{e}-E_{0}}
$$

involving all possible excited states $|e\rangle$, we follow a derivation given by Ferrell for the analogous case of Josephson tunneling between two BCS superconductors [26. As is evident from (9), there are three contributions to the transfer Hamiltonian involving excited states: two c-nc terms and one nc-nc contribution. Denoting time reversed states by $\bar{k}=-k$ and using the time reversal invariance relation $t_{c k}=t_{\bar{k} c}$, the contribution which describes tunneling between condensate $b$ and noncondensate states in $a$ can be written as

$$
\hat{H}_{T}^{c_{b}-n c_{a}}=-\sum_{k} t_{k c}\left(\hat{a}_{k}^{\dagger} \hat{a}_{c b}+\hat{a}_{c b}^{\dagger} \hat{a}_{\bar{k}}\right) .
$$

Starting in a state $|\nu\rangle$ with a definite number of atoms in each condensate but no excitations, the relevant excited states $|k, \nu\rangle$ associated with the first term in (11) are those, in which a condensate atom from $b$ is transferred to a quasiparticle $k$ in $a$. With the standard Bogoliubov transformation

$$
\hat{a}_{k}^{\dagger}=u_{k} \hat{\alpha}_{k}^{\dagger}-v_{k} \hat{\alpha}_{\bar{k}}
$$

between the Boson or quasiparticle creation operators $\hat{a}_{k}^{\dagger}$ or $\hat{\alpha}_{k}^{\dagger}$, the associated matrix element is 


$$
\left\langle k, \nu\left|\hat{H}_{T}^{c_{b}-n c_{a}}\right| \nu\right\rangle=-t_{k c} u_{k} \sqrt{\bar{N}_{b}},
$$

again assuming $|\nu| \ll \bar{N}_{b}$. Now precisely the same excited state with energy $E_{k}$ (both $E_{k}$ and the particle and hole amplitudes $u_{k}$ and $v_{k}$ are positive and even in $k$, following standard notation [27]) can be reached from the state $|\nu+2\rangle$ by converting two condensate atoms in $a$ to a pair $(k, \bar{k})$ and transferring one of the partners $\bar{k}$ to a condensate state in $b$, as indicated by the second term in (9). The amplitude for this process is

$$
\left\langle k, \nu\left|\hat{H}_{T}^{c_{b}-n c_{a}}\right| \nu+2\right\rangle=t_{k c} v_{k} \sqrt{\bar{N}_{b}} .
$$

It differs from (13) by the replacement $u_{k} \rightarrow-v_{k}$ since the amplitude for creating a quasiparticle with momentum $k$ by destroying an atom with momentum $-k$ is $-v_{k}$. In more physical terms, the relative minus sign between these amplitudes may be understood by noting, that in the Bogoliubov theory (and in fact quite generally for a Bose condensed system) there is a fixed relative phase $\pi$ between the condensate and pairs $(k, \bar{k})$ of noncondensate atoms 28]. This minimizes the repulsive interaction, giving rise to a gapless excitation spectrum in contrast to the result of a Hartree-Fock approximation. The phase locking between condensate and noncondensate is also implicit in the form

$$
\left|\varphi_{a}\right\rangle \propto \exp \left(e^{i \varphi_{a}} \sqrt{N_{a}} \hat{a}_{c, a}^{\dagger}-e^{2 i \varphi_{a}} \sum_{k} \frac{u_{k}}{v_{k}} \hat{a}_{k}^{\dagger} \hat{a}_{\bar{k}}^{\dagger}\right)
$$

of the Bogoliubov ground state for a homogeneous system with a well defined overall phase $\varphi_{a}$, as introduced in (4) above. Now, as emphasized before, the ground state of the coupled condensates is one with a definite relative phase $\varphi$, in which the amplitudes (13) and (14) add coherently

$$
\left\langle k, \nu\left|\hat{H}_{T}^{c_{b}-n c_{a}}\right| \varphi\right\rangle=-e^{i \nu \varphi} t_{k c} \sqrt{\bar{N}_{b}}\left(u_{k}-e^{2 i \varphi} v_{k}\right) .
$$

With $E_{k}$ as the relevant excitation energy, this gives rise to a phase dependent contribution

$$
\bar{N}_{b} \sum_{k} \frac{\left|t_{k c}\right|^{2}}{E_{k}} 2 u_{k} v_{k} \cdot \cos 2 \varphi
$$

to the second order energy shift. Using $2 u_{k} v_{k}=\mu_{a} / E_{k}$ and exchanging the roles of condensates $a$ and $b$, the total c-nc contribution to the phase dependent energy in second order is

$$
\left(\bar{N}_{a} \mu_{b} \sum_{q} \frac{\left|t_{c q}\right|^{2}}{E_{q}^{2}}+\bar{N}_{b} \mu_{a} \sum_{k} \frac{\left|t_{k c}\right|^{2}}{E_{k}^{2}}\right) \cdot \cos 2 \varphi .
$$

Obviously this term, which is much larger than the nc-nc contribution derived below, favors a relative phase $\varphi=$ $\pi / 2$ of the coupled condensates. In the weak tunneling regime considered here, however, it is always the leading first order term (7) which dominates, leading to a ground state with $\varphi=0$. To obtain the nc-nc contribution, we use again time reversal invariance to write

$$
\hat{H}_{T}^{n c-n c}=-\sum_{k, q} t_{k q}\left(\hat{a}_{k}^{\dagger} \hat{a}_{q}+\hat{a}_{\bar{q}}^{\dagger} \hat{a}_{\bar{k}}\right) .
$$

The relevant excited states $|k, \bar{q}, \nu\rangle$ now have energy $E_{k}+$ $E_{q}$ and are characterized by $\bar{N}_{a}+\nu$ and $\bar{N}_{b}-\nu-2$ atoms in condensates $a$ and $b$ plus one quasiparticle $k$ respectively $\bar{q}$ on each side. Corresponding to the two contributions in (19), these states can be reached starting from either $|\nu\rangle$ or $|\nu+2\rangle$. Adding the respective amplitudes in the actual coherent ground state with a well defined relative phase, one obtains

$$
\left\langle k, \bar{q}, \nu\left|\hat{H}_{T}^{n c-n c}\right| \varphi\right\rangle=e^{i \nu \varphi} t_{k q}\left(u_{k} v_{q}+e^{2 i \varphi} v_{k} u_{q}\right) .
$$

The phase dependent part of the associated second order energy shift is thus

$$
\Delta E_{n c-n c}^{(2)}(\varphi)=-2 \sum_{k, q}\left|t_{k q}\right|^{2} \frac{u_{k} v_{k} u_{q} v_{q}}{E_{k}+E_{q}} \cdot \cos 2 \varphi,
$$

in perfect analogy to the result obtained for a superconducting Josephson contact [24,26], where this is the only contribution. Using the relation between the average of $\left|t_{k q}\right|^{2}$ at the Fermi energy and the normal state conductance, (21) directly leads to the result (8) at zero temperature. For weakly interacting Bose gases, in turn, with the explicit results (53) and (54) for the matrix elements given below, the contribution (21) is easily shown to be smaller than the c-nc contribution (18) by a factor $\sqrt{n a^{3}}$ [29], and thus is negligible for gaseous BECs.

For a given static value $\varphi$ of the phase difference between both condensates, the nondissipative current obtained from (6) up to second order in $\hat{H}_{T}$ is thus of the form

$$
I(\varphi)=-I_{c} \sin \varphi-J_{1}(0) \sin 2 \varphi,
$$

with a positive critical current $I_{c}=E_{J} / \hbar$. The magnitude $J_{1}(0)<0$ of the second order contribution is $-2 / \hbar$ times the factor in parentheses in (18). The small second order term changes the value of $\varphi$ at which the current is maximal from $\pi / 2$ to $\pi / 2+2\left|J_{1}(0)\right| / I_{c}$, however the critical current itself is unchanged to this order. In an open system, with a reservoir of particles, where it is possible to impose a constant external current, the phase difference adjusts itself to a constant value, determined by (22). This is the well known dc-Josephson effect, i.e. a finite current at vanishing chemical potential difference $\Delta \mu=0$. In the case of two coupled BECs with a fixed total number of particles, any current flow is connected with a finite value of $\Delta \mu$ even in the absence of an external potential drop, because $\mu$ depends on the particle number. Thus, by the Josephson relation (23), one has inevitably a phase difference which evolves in time. In addition, finite dissipative currents appear, requiring a fully dynamical treatment, as will be given in the following sections. 


\section{JOSEPHSON AND NORMAL CURRENTS AT GIVEN $\Delta \mu$}

In the following, we want to determine the complete current in a situation with a finite difference in the chemical potentials. We start by considering the idealized case in which $\Delta \mu$ is considered as a fixed, externally given value. This approximation, which applies to open systems like standard Josephson contacts between superconductors, neglects the change in the chemical potential associated with the transfer of particles in a system with a fixed total number of particles, an effect which is taken up in section 5. A finite value of $\Delta \mu$ gives rise to an additional term $\Delta \mu \hat{\nu}$ to the total Hamiltonian (1). In close analogy to the calculation of currents in normal and superconducting tunnel junctions, this term may formally be eliminated by a time dependent gauge transfomation. One thus obtains a tunneling Hamiltonian with $\Delta \mu=0$, in which the transfer matrix elements are modulated in time with a factor $e^{i \varphi(t)}$ such that

$$
\hbar \frac{d}{d t} \varphi(t)=-\Delta \mu \text {. }
$$

Since the current operator

$$
\hat{I}=-\frac{d}{d t} \hat{N}_{a}=i\left(\hat{\Lambda}-\hat{\Lambda}^{\dagger}\right) / \hbar
$$

is already linear in the tunneling matrix elements, the problem can, up to second order in $\hat{H}_{T}$, be formally treated like in time dependent linear response 20]. It is then straightforward to show that the expectation value $I$ of the current is given by

$$
\begin{gathered}
I=\frac{i}{\hbar}\left\langle e^{-i \varphi(t)} \hat{\Lambda}(t)-e^{i \varphi(t)} \hat{\Lambda}^{\dagger}(t)\right\rangle \\
+\frac{2}{\hbar^{2}} \Re \int_{-\infty}^{t} d t^{\prime}\left\{e^{-i\left(\varphi(t)-\varphi\left(t^{\prime}\right)\right)}\left\langle\left[\hat{\Lambda}(t), \hat{\Lambda}^{\dagger}\left(t^{\prime}\right)\right]\right\rangle\right. \\
\left.+e^{-i\left(\varphi(t)+\varphi\left(t^{\prime}\right)\right)}\left\langle\left[\hat{\Lambda}(t), \hat{\Lambda}\left(t^{\prime}\right)\right]\right\rangle\right\},
\end{gathered}
$$

where the time dependence of the operators $\hat{\Lambda}$ and $\hat{\Lambda}^{\dagger}$ has to be taken with respect to the unperturbed Hamiltonian $\hat{H}_{a}+\hat{H}_{b}$. The expectation values reduce to ones in the associated ground state, provided we restrict ourselves to the limit of zero temperature, as is done throughout in the following.

As was pointed out above, the eigenstates of $\hat{H}_{a}+\hat{H}_{b}$ for gaseous BECs can be choosen as the Gross-Pitaevski wave functions in each well, and a set of excited states obtained from solving the Bogoliubov equations in local density approximation. As long as the number of transferred particles remains small compared to $\bar{N}_{a / b}$, these states are unaffected to lowest order. Thus both the condensate wave function as well as the quasiparticle energies $E_{k}$ and amplitudes $u_{k}$ and $v_{k}$ are unchanged by the current flow. The comparison with the numerical results in section 7 will show, that at least on the GrossPitaevski level this approximation is well justified in the weak tunneling limit discussed here.
The first term on the right hand side of Eq. (25) is then

$$
\begin{aligned}
& \frac{i}{\hbar}\left\langle e^{-i \varphi(t)} \hat{\Lambda}(t)-e^{i \varphi(t)} \hat{\Lambda}^{\dagger}(t)\right\rangle \\
& \quad=-\frac{2 t_{c c}}{\hbar} \sqrt{\bar{N}_{a}^{c} \bar{N}_{b}^{c}} \sin \varphi(t)=-I_{c} \sin \varphi(t),
\end{aligned}
$$

in perfect agreement with the result derived in the previous section for a static situation. The second term in Eq. (25) involves noncondensate tunneling. At fixed $\Delta \mu$, it is readily evaluated within Bogoliubov theory, giving three contributions:

$$
\begin{gathered}
\frac{2}{\hbar^{2}} \Re \int_{-\infty}^{t} d t^{\prime}\left\{e^{-i\left(\varphi(t)-\varphi\left(t^{\prime}\right)\right)}\left\langle\left[\hat{\Lambda}(t), \hat{\Lambda}^{\dagger}\left(t^{\prime}\right)\right]\right\rangle\right. \\
\left.+e^{-i\left(\varphi(t)+\varphi\left(t^{\prime}\right)\right)}\left\langle\left[\hat{\Lambda}(t), \hat{\Lambda}\left(t^{\prime}\right)\right]\right\rangle\right\} \\
=J_{n}(\Delta \mu)-J_{1}(\Delta \mu) \sin 2 \varphi(t)+J_{2}(\Delta \mu) \cos 2 \varphi(t) .
\end{gathered}
$$

The first one is a phase independent normal current, accounting for the expected dissipative current flow towards the lower chemical potential. The $\sin 2 \varphi$-term describes a second order Josephson current, associated with the corresponding phase dependent coupling energy derived in the previous section. Finally there is an interference current proportional to $\cos 2 \varphi$ which is out of phase by $\pi / 2$ from the corresponding Josephson contribution. Similar to the related $\cos \varphi$-term in superconductors [11], this is a dissipative current which vanishes at $\Delta \mu=0$, as the normal current does. Within the local density approximation for solving the Bogoliubov-equations, the current amplitudes $J_{n}, J_{1}$ and $J_{2}$ can be expressed in terms of the tunneling matrix elements and the normal and anomalous spectral functions $A(k, \omega)$ and $B(k, \omega)$ of the weakly interacting homogeneous Bose gas. In standard notation [27] they read

$$
\begin{aligned}
& A(k, \omega)=2 \pi\left\{u_{k}^{2} \delta\left(\omega-E_{k} / \hbar\right)-v_{k}^{2} \delta\left(\omega+E_{k} / \hbar\right)\right\} \\
& B(k, \omega)=2 \pi u_{k} v_{k}\left\{\delta\left(\omega+E_{k} / \hbar\right)-\delta\left(\omega-E_{k} / \hbar\right)\right\} .
\end{aligned}
$$

With $n_{B}(x)=(\exp \beta x-1)^{-1}$ as the Bose distribution function, the current amplitudes in Eq. (27) are then given by

$$
\begin{aligned}
& J_{n}(\Delta \mu)=\bar{N}_{a}^{c} \sum_{q} \frac{\left|t_{c q}\right|^{2}}{\hbar^{2}} A(q, \Delta \mu / \hbar) \\
& -\bar{N}_{b}^{c} \sum_{k} \frac{\left|t_{k c}\right|^{2}}{\hbar^{2}} A(k,-\Delta \mu / \hbar) \\
& +\sum_{k, q} \frac{\left|t_{k q}\right|^{2}}{\hbar^{2}} \int_{-\infty}^{\infty} \frac{d \omega}{2 \pi}\left[n_{B}(\hbar \omega)-n_{B}(\hbar \omega+\Delta \mu)\right] \\
& A(k, \omega) A(q, \omega+\Delta \mu / \hbar)
\end{aligned}
$$

and 


$$
\begin{gathered}
J_{2}(\Delta \mu)=\bar{N}_{a}^{c} \sum_{q} \frac{\left|t_{c q}\right|^{2}}{\hbar^{2}} B(q,-\Delta \mu / \hbar) \\
+\bar{N}_{b}^{c} \sum_{k} \frac{\left|t_{k c}\right|^{2}}{\hbar^{2}} B(k,-\Delta \mu / \hbar) \\
-\sum_{k, q} \frac{\left|t_{k q}\right|^{2}}{\hbar^{2}} \int_{-\infty}^{\infty} \frac{d \omega}{2 \pi}\left[n_{B}(\hbar \omega)-n_{B}(\hbar \omega+\Delta \mu)\right] \\
B(k, \omega) B(q, \omega+\Delta \mu / \hbar) .
\end{gathered}
$$

The amplitude $J_{1}(\Delta \mu)$ of the second order Josephson current can be obtained from that of the associated dissipative contribution $J_{2}(\Delta \mu)$ via a Kramers-Kronig relation

$$
J_{1}(\Delta \mu)=-\mathcal{P} \int_{-\infty}^{\infty} \frac{d \omega}{\pi} \frac{J_{2}(\hbar \omega)}{\omega-\Delta \mu / \hbar} .
$$

It is straightforward to check, that at zero temperature and vanishing $\Delta \mu$, the combination $\hbar J_{1}(0) / 2$ is identical with the sum of the coefficients of $\cos 2 \varphi$ in (18) and (21) of the second order phase dependent coupling energy, as it should. As noted in the previous section, a particular feature of tunneling between Bose condensates is the appearance of c-nc contributions. Indeed, as the first two terms in Eq. (30) show, they also contribute to the phase independent normal current, where they are in fact dominant compared to the conventional nc-nc contribution described by the last term. Another point that should be mentioned, is the absence of a second order contribution due to c-c tunneling [29]. This follows because the commutators involving only condensate operators in Eq. (25) vanish. A different proof of this result may be obtained, by considering all possible Feynman diagrams associated with second order tunneling processes. Diagrams proportional to $\left|t_{c c}\right|^{2}$ are reducible and thus give no separate contribution.

The similarity of the second order currents in BECs and superconductors is a result of the close formal analogy of Bogoliubov and BCS theory. The additional contributions due to c-nc tunneling in BECs lead to interesting new features, however. As will be discussed in section 6 , for the Bose case the dissipative currents $J_{n}(\Delta \mu)$ and $J_{2}(\Delta \mu)$ remain finite at zero temperature in contrast to superconductors, where they vanish exponentially due to the presence of an energy gap. These currents thus provide a mechanism for the damping of Josephson oscillations even at $T=0$. Similar to the situation in superconductors [30], it may be shown that the total dissipation associated with the two irreversible currents is always positive [29]. Physically, this property is equivalent to the statement, that the sum $J_{n}+J_{2} \cos 2 \varphi$ is strictly positive for $\Delta \mu>0$, i.e. the total dissipative current always flows towards lower chemical potential. It therefore leads to a reduction of $\Delta \mu$ and hence to a damping of the phase dynamics, similar to the contribution of excited states in driven two component condensates discussed in 18 .

\section{PATH INTEGRAL DESCRIPTION}

So far, we assumed $\hbar \dot{\varphi}$ to be given externally, thus neglecting the variation of the chemical potential with the total number of particles. As we will see, however, this effect is quantitatively important even in the regime where $N_{a}-N_{b} \ll \bar{N}_{a / b}$. To lowest order in $\nu / \bar{N}_{a / b}$, the dependence of the chemical potential on the actual number of particles can be accounted for by an additional 'charging' energy $U\left(\hat{N}_{a}-\hat{N}_{b}\right)^{2} / 8$, with $U=\partial \mu_{a} / \partial N_{a}+\partial \mu_{b} / \partial N_{b}$ being of order $\mu / \bar{N}$. In the limit $U \gtrsim \hbar I_{c}$, this term suppresses phase coherence, since by canonical quantization $\hat{\nu} \rightarrow i \partial / \partial \varphi$, charging effects are equivalent to quantum fluctuations of $\varphi$. For any $U \neq 0$, the chemical potential difference in $\hbar \dot{\varphi}=-\Delta \mu$ contains an internal contribution $U \nu$ proportional to the number of transferred bosons. The resulting dynamics of $\varphi$ is therefore intrinsically nonlinear. Within a simple description, these effects have already been introduced by Smerzi et.al. [16], who also took into account the $\nu$-dependence of the Josephson coupling $E_{J} \propto \sqrt{\left(\bar{N}_{a}+\nu\right)\left(\bar{N}_{b}-\nu\right)}$. While this effect is indeed relevant for strong asymmetries $\nu=O\left(\bar{N}_{a / b}\right)$, it is negligible compared to the charging term of order $\mu \nu^{2} / \bar{N}$ provided we are in the regime $E_{J} \ll \mu \bar{N}$ where Josephson tunneling is a small perturbation. In order to include charging effects in a microscopic description, we use the formalism developed by Ambegaokar, Eckern and Schön [31] for superconductor tunnel junctions.

In a coherent state path integral formulation for interacting bosons, the grand- canonical partition function of two coupled condensates is given by

$$
\begin{aligned}
Z=\int & D \psi_{a} D \psi_{b} \exp -\int_{0}^{\beta \hbar} \frac{d \tau}{\hbar}\left[\int d^{3} x \psi_{a}^{*}\left(\hbar \partial_{\tau}-\mu_{a}\right) \psi_{a}\right. \\
& \left.+\int d^{3} y \psi_{b}^{*}\left(\hbar \partial_{\tau}-\mu_{b}\right) \psi_{b}+H\left(\psi_{a}, \psi_{b}\right)\right] .
\end{aligned}
$$

Here $\mu_{a / b}$ fix the average number $\bar{N}_{a / b}$ of atoms in each condensate, while $H\left(\psi_{a}, \psi_{b}\right)$ is the standard representation of the basic Hamiltonian (1), in which the bosonic field operators are replaced by complex c-number fields $\psi_{a / b}(x / y, \tau)$, depending both on the spatial coordinates plus an imaginary time like variable $\tau$ [32]. With the charging term explicitly included in $H\left(\psi_{a}, \psi_{b}\right)$, Eq. (33) is also appropriate for describing a system with $N_{a}+N_{b}$ fixed. As in the analogous fermionic problem of coupled superconductors, it is convenient to introduce an auxiliary path integral $\int D V$ to remove the charging term quartic in the fields via a Hubbard-Stratonovich transformation. The functional integral is then evaluated in a saddle point approximation, the saddle point being just the condensate wavefunction for two separate condensates. Performing a gauge transformation, we may replace the integration variable $V(\tau)$ by the phase $\varphi(\tau)$, which is the dynamical variable of interest. Furthermore, we introduce Nambu spinors $\tilde{\Psi}^{\dagger}=\left(\tilde{\psi}_{a}^{*}, \tilde{\psi}_{a}, \tilde{\psi}_{b}^{*}, \tilde{\psi}_{b}\right)$ for the Gaussian fluctuations around the saddle point 
$\Phi^{\dagger}=\left(\phi_{a}^{*}, \phi_{a}, \phi_{b}^{*}, \phi_{b}\right)$. The full partition function is then given by

$$
\begin{aligned}
Z= & e^{-S_{0}} \int D \varphi \int D \tilde{\Psi} D \tilde{\Psi}^{\dagger} \\
& \exp -\int_{0}^{\beta \hbar} \frac{d \tau}{\hbar}\left[\frac{\hbar^{2} \dot{\varphi}^{2}}{2 U}-E_{J} \cos \varphi\right. \\
+ & \left.\frac{1}{2} \int d^{3} x d^{3} y \tilde{\Psi}^{\dagger}\left(-\underline{G}^{-1}+\tilde{t}\right) \tilde{\Psi}+\tilde{\Psi}^{\dagger} \tilde{t} \Phi+\Phi^{\dagger} \tilde{t} \tilde{\Psi}\right],
\end{aligned}
$$

where $S_{0}$ is a bulk contribution to the action. In coordinate space, the $4 \times 4$ tunneling matrix $\tilde{t}$ is defined by

$$
\tilde{t}=\left(\begin{array}{cc}
0 & \hat{t}_{x y} \\
\hat{t}_{x y}^{\dagger} & 0
\end{array}\right), \quad \hat{t}=\left(\begin{array}{cc}
t_{x y} & 0 \\
0 & t_{x y}^{*}
\end{array}\right)
$$

while the differential operator $\underline{G}^{-1}$ satisfies

$$
\underline{G}^{-1}\left(\begin{array}{cc}
\underline{G}_{a} & 0 \\
0 & \underline{G}_{b}
\end{array}\right)=\left(\begin{array}{cc}
\delta\left(x-x^{\prime}\right) \underline{1} & 0 \\
0 & \delta\left(y-y^{\prime}\right) \underline{1}
\end{array}\right) \delta\left(\tau-\tau^{\prime}\right) .
$$

In the last equation, $\underline{G}_{a / b}$ is shorthand for the noncondensate part of the $2 \times 2$ matrix Green's function for a weakly interacting Bose gas, as defined in [27].

Completing the square in the exponent of (34), the Gaussian integral over $\tilde{\Psi}$ is readily performed and yields

$$
Z=Z_{a} Z_{b} \int D \varphi(\tau) \exp (-S[\varphi] / \hbar) .
$$

Here, $Z_{a / b}$ are the partition functions of the separate condensates, while tunneling effects are taken into account by an effective action for the relative phase

$$
\begin{array}{r}
S[\varphi]=\int_{0}^{\beta \hbar} d \tau\left(\frac{\hbar^{2} \dot{\varphi}^{2}}{2 U}-E_{J} \cos \varphi\right) \\
-\hbar \int_{0}^{\beta \hbar} d \tau d \tau^{\prime}\left[\alpha\left(\tau-\tau^{\prime}\right) \cos \left(\varphi-\varphi^{\prime}\right)\right. \\
\left.+\beta\left(\tau-\tau^{\prime}\right) \cos \left(\varphi+\varphi^{\prime}\right)\right]
\end{array}
$$

where $\varphi=\varphi(\tau)$ and $\varphi^{\prime}=\varphi\left(\tau^{\prime}\right)$. The integral kernels $\alpha(\tau)$ and $\beta(\tau)$ may be expressed in terms of the currents $J_{n}(\Delta \mu)$ and $J_{2}(\Delta \mu)$, introduced in the previous section via their Fourier coefficients

$$
\begin{aligned}
& \alpha\left(i \omega_{n}\right)=\int_{-\infty}^{\infty} \frac{d \omega}{2 \pi} \frac{J_{n}(-\hbar \omega)}{i \omega_{n}-\omega} \\
& \beta\left(i \omega_{n}\right)=\int_{-\infty}^{\infty} \frac{d \omega}{2 \pi} \frac{J_{2}(\hbar \omega)}{i \omega_{n}-\omega},
\end{aligned}
$$

in an expansion of the $\beta \hbar$-periodic functions

$$
\alpha(\tau)=\frac{1}{\beta \hbar} \sum_{n} e^{-i \omega_{n} \tau} \alpha\left(i \omega_{n}\right)
$$

in bosonic Matsubara frequencies $\omega_{n}=2 \pi n / \beta \hbar, n$ integer.

The effective action Eq. (38) is formally identical with the one obtained for a superconducting Josephson contact [31], except for the additional local contribution $-E_{J} \cos \varphi(\tau)$ describing the first order Josephson effect due to c-c tunneling. The terms nonlocal in time give rise to both the second order Josephson currents related to $\beta\left(\tau-\tau^{\prime}\right)$ and the normal current associated with $\alpha\left(\tau-\tau^{\prime}\right)$. For the idealized model discussed in section 6 , where $J_{2}(\Delta \mu)$ and $J_{n}(\Delta \mu)$ are linear for small values of the chemical potential difference, both $\alpha(\tau)$ and $\beta(\tau)$ asymptotically decay like $1 / \tau^{2}$, i.e., the Josephson contact exhibits ohmic dissipation.

An exact evaluation of the path integral in (37) is obviously impossible, since the effective action is nonlocal and - in particular - nongaussian. Nevertheless, as shown by Ambegaokar, Eckern and Schön [31], it is a useful starting point for the derivation of a semiclassical approximation for the phase dynamics. Indeed, from the imaginary time description, it is possible to derive a semiclassical equation of motion in real time, which has the form of a classical Langevin equation with state dependent quantum noise. In the limit where $J_{2}(\Delta \mu)$ and the normal current $J_{n}(\Delta \mu)=G_{n} \Delta \mu$ are purely ohmic, it reads

$$
\frac{\hbar \ddot{\varphi}}{U}+G(\varphi) \hbar \dot{\varphi}+I_{c} \sin \varphi+J_{1} \sin 2 \varphi=\eta_{1} \cos \varphi+\eta_{2} \sin \varphi,
$$

where $\eta_{1,2}$ are independent Gaussian random forces. Their autocorrelation function may be expressed in terms of the real time functions $\alpha(t)$ and $\beta(t)$ via

$$
\left\langle\eta_{1 / 2}(t) \eta_{1 / 2}\left(t^{\prime}\right)\right\rangle=2\left[\alpha^{I}\left(t-t^{\prime}\right) \mp \beta^{I}\left(t-t^{\prime}\right)\right],
$$

where $\alpha^{I}$ and $\beta^{I}$ are the imaginary parts of the analytic continuation of $\alpha(\tau)$ and $\beta(\tau)$ similar to [31]. The damping term is proportional to a phase dependent effective conductance $G(\varphi)=G_{n}(1+\cos 2 \varphi)$, with a normal conductance $G_{n}$, which will be explicitly evaluated in section 6 . It gives rise to a relaxational dynamics with a typical time scale $1 / G_{n} U$. Even without damping, however, the $\ddot{\varphi}$-term leads to currents which are not perfectly sinusoidal for a given external chemical potential difference. Neglecting the fluctuating forces, the resulting average dynamics will be discussed in section 7 for realistic values of the parameters in weakly coupled BECs.

\section{AN EXPLICIT MODEL}

If the trap potential close to the barrier varies slowly on the scale of the coherence length $\xi$, we may apply the approximations discussed in section 3, using eigenstates of a locally homogeneous system with constant external potential throughout $a$ and $b$, respectively. A simple model geometry for such a Josephson junction is shown in Fig. 1: 


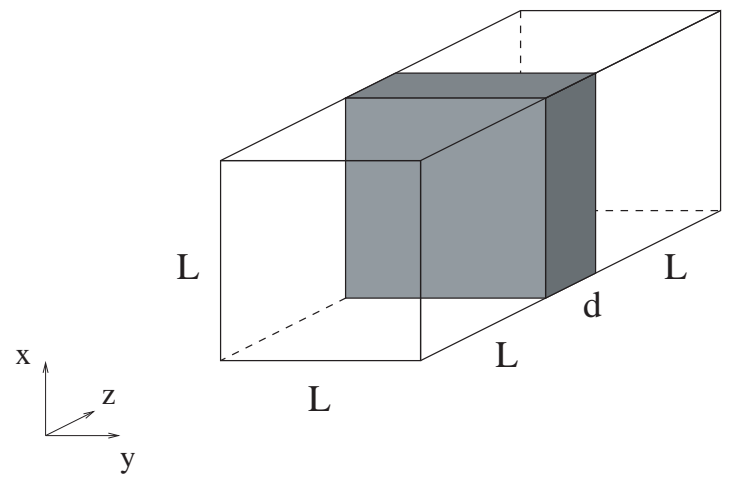

FIG. 1. The model geometry considered for the evaluation of the current densities.

Two BECs confined in a cubic volume $L^{3}$ are separated by a square potential barrier of height $V_{B}$ and width $d$. For simplicity, we impose periodic boundary conditions in the directions parallel to the potential barrier. This simple model accounts for the fact, that all currents must scale linearly with area. It allows us to calculate the relevant currents per area in terms of microscopic parameters of the bulk condensates. For a concrete experimental setup, one may then determine the actual critical current $I_{c}=j_{c} A$ from the associated current densities and the effective contact area $A$.

For this system, the currents $I_{c}, J_{n}, J_{1}$ and $J_{2}$ may be evaluated explicitly from the detailed form of the tunneling matrix elements. Since we have assumed translation invariance parallel to the barrier, the associated momenta $\vec{k}_{\|}, \vec{q}_{\|}$are conserved, and the problem is effectively one-dimensional (1d). The tunneling amplitudes are then obtained from the current matrix elements [20]

$$
t_{l r}=\frac{\hbar^{2}}{2 m}\left[\chi_{l} \frac{d \chi_{r}}{d z}-\chi_{r} \frac{d \chi_{l}}{d z}\right]_{z=0} \cdot \delta_{\vec{k}_{\|}, \vec{q}_{\|}}
$$

of the $1 \mathrm{~d}$ wave functions $\chi_{l, r}(z)$, taken at the center of the barrier at $z=0$. In the limit of weak tunneling, the barrier height $V_{B}$ is much larger than the average chemical potential $\bar{\mu}=\left(\mu_{a}+\mu_{b}\right) / 2$. The wave functions in (44) are therefore effectively single particle eigenstates which decay exponentially like $\exp \pm \kappa_{\mu} z$, with inverse characteristic length $\kappa_{\mu}=\sqrt{2 m\left(V_{B}-\mu\right) / \hbar^{2}}$. For a small difference $\Delta \mu=\mu_{a}-\mu_{b} \ll \bar{\mu}$ in the chemical potentials, (44) then reduces to

$$
t_{l r}=\frac{\hbar^{2} \kappa_{\bar{\mu}}}{m} \chi_{l}(0) \chi_{r}(0) \cdot \delta_{\vec{k}_{\|}, \vec{q}_{\|}} .
$$

The calculation of the different tunneling amplitudes $t_{c c}$, $t_{k c}$ and $t_{k q}$, thus requires

- a solution of the 1d GPE for a finite barrier, which approaches $\phi_{c}=\sqrt{N_{c}} \cdot \chi_{c} \rightarrow \sqrt{n_{c}}$ far from the barrier, i.e. a uniform condensate with density $n_{c}$ (note that the boundaries at $z= \pm(L+d / 2)$ are irrelevant since $L \gg \xi$ ), and
- corresponding solutions of the 1d Bogoliubov equations with finite momenta $k, q$, which smoothly connect to the exponentially decaying single particle states below the barrier.

For concreteness we consider the eigenstates $\chi_{l}$ in condensate $a$, to the left of the barrier. It is then straightforward to show that

$$
\begin{aligned}
\chi_{c, a}(z)= & \frac{1}{\sqrt{L}} \frac{\kappa_{\mu_{a}} \xi_{a}}{\sqrt{2}}\left(\sqrt{1+\frac{2}{\left(\kappa_{\mu_{a}} \xi_{a}\right)^{2}}}-1\right) e^{-\kappa_{\mu_{a}}(z+d / 2)} \\
& \stackrel{V_{B} \gg \mu_{a}}{\longrightarrow} \frac{1}{\sqrt{L}} \frac{1}{\sqrt{2} \kappa_{\mu_{a}} \xi_{a}} e^{-\kappa_{\mu_{a}}(z+d / 2)}
\end{aligned}
$$

provides an appropriate solution of the GPE. It is obtained by connecting the well known solution $-\sqrt{n_{c}} \tanh \frac{z+\delta}{\sqrt{2} \xi}$ of the full GPE in the regime $z<-d / 2$ to an exponentially decaying solution of the linearized equation below the barrier $z>-d / 2$. The condition that $\chi$ and its first derivative are continuous at $z=-d / 2$ fixes the prefactor in the solution of the linear equation. The linearization is justified in the limit $\kappa \xi \gg 1$, where the mean field interaction is negligible compared to the repulsive potential separating the two condensates. Using the solution (46) and the condition $\Delta \mu \ll \bar{\mu}$, the c-c tunneling amplitude is given by

$$
t_{c c}=f\left(V_{B} / \bar{\mu}\right) \cdot \frac{\sqrt{\mu_{a} \mu_{b}}}{\kappa_{\bar{\mu}} L} e^{-\kappa_{\bar{\mu}} d},
$$

with a correction factor

$$
f(x)=\left(1-\left(x-\sqrt{x^{2}-1}\right)\right)^{2},
$$

which is smaller than one and approaches unity in the high barrier limit $V_{B} \gg \bar{\mu}$. Since $t_{c c}$ is proportional to $1 / L$, the resulting Josephson coupling energy $E_{J}=$ $2 t_{c c} \sqrt{\bar{N}_{a} \bar{N}_{b}}$ scales like the contact area $A=L^{2}$, as it should. The associated critical current density is

$$
j_{c}=2 f\left(V_{B} / \bar{\mu}\right) \frac{\left(\mu_{a} n_{c, a} \mu_{b} n_{c, b}\right)^{1 / 2}}{\hbar \kappa_{\bar{\mu}}} \cdot e^{-\kappa_{\bar{\mu}} d} .
$$

As expected, it is linear in the barrier transmission amplitude $\exp -\kappa_{\bar{\mu}} d$. More interesting is the prefactor which, by the standard relation $2 p=\mu n_{c}$ for the ground state pressure of a weakly interacting Bose gas, is just proportional to the geometric average of the ground state pressures $p_{a}$ and $p_{b}$ of the two condensates. It is instructive to compare this with the corresponding result for coupled ideal Bose gases, where the condensate wave functions are the well known wavefunctions of the one particle ground state in a potential well of finite height. The associated c-c tunneling amplitude is then readily evaluated, giving

$$
t_{c c}^{(0)}=2 \pi^{2} \frac{\hbar^{2}}{m} \frac{1}{\kappa_{\bar{\mu}} L^{3}} e^{-\kappa_{\bar{\mu}} d} .
$$


¿From this, we immediately find a critical current

$$
I_{c}^{(0)}=4 \pi^{2} \frac{\hbar \sqrt{\bar{n}_{a}^{c} \bar{n}_{b}^{c}}}{m \kappa_{\bar{\mu}}} e^{-\kappa_{\bar{\mu}} d}
$$

which is independent of the contact area. Evidently, the behaviour implied by Eq. (51) is an artefact of the ideal Bose gas, which exhibits a vanishing quantum pressure below the condensation temperature $T_{c}$ in the thermodynamic limit. The critical current density thus remains finite for large contact areas only if the repulsive interaction between the particles is included. The linear scaling of the critical current with area is also found in the model by Zapata et al. [17]. In fact, our result for the critical current agrees with theirs for a plane barrier to leading order in $e^{-\kappa_{\bar{\mu}} d}$, i.e. with $f=1$. As a final point of our discussion of c-c tunneling, we mention that due to the $N$ dependence of the chemical potential in (47), the amplitude $t_{c c}$ - which is identical with the coupling parameter $K$ introduced in [16] - depends on the number of particles in the condensate. In the limit $|\nu| \ll \bar{N}_{a / b}$ discussed here, this effect is negligible compared to the charging effects, as mentioned above. In situations, however, where $|\nu|$ becomes itself of order $N_{a / b}$, this dependence is important and the c-c tunneling amplitude cannot be treated as a constant, as assumed in ref. 16].

To calculate the remaining amplitudes $t_{k c}$ and $t_{k q}$ which enter into the higher order and dissipative currents, we need to match a solution of the $1 \mathrm{~d}$ Bogoliubov equation for a homogeneous condensate in $z<-d / 2$ to an exponentially decaying wave function below the barrier. Since the condensate density $n_{c}(z)$ there vanishes very quickly like exp $-2 \kappa z$, the mixing between the amplitudes $u_{k}(z)$ and $v_{k}(z)$ may be neglected (for simplicity, $k$ and $q$ here are just the $z$-components of the original $3 \mathrm{~d}$ wave vectors $k$ and $q$ ). Connecting the trivial solution $(2 / L)^{1 / 2} \sin (k z+\delta)$ for $u_{k}(z)$ in $a$, to one below the barrier such that the function and its first derivative are continuous at $z=-d / 2$, we find

$$
\chi_{k}(z)=\left(\frac{2}{L}\right)^{1 / 2} \frac{k}{\kappa_{\mu_{a}}} \cdot e^{-\kappa_{\mu_{a}}(z+d / 2)} .
$$

Here, it has been assumed that $k \ll \kappa$, i.e. the kinetic energy for motion perpendicular to the barrier is small compared to $V_{B}$. The tunneling amplitudes involving noncondensate states now follow easily from (45) and are given by

$$
t_{k c}=\frac{\hbar^{2}}{m \xi_{a} \kappa_{\bar{\mu}} L} \frac{k}{\sqrt{1+(k \xi)^{2}}} e^{-\kappa_{\bar{\mu}} d} \cdot \delta_{\vec{k}_{\|}, \overrightarrow{0}}
$$

and

$$
t_{k q}=\frac{2 \hbar^{2}}{m \kappa_{\bar{\mu}} L} \frac{k q}{\sqrt{1+(k \xi)^{2}} \sqrt{1+(q \xi)^{2}}} e^{-\kappa_{\bar{\mu}} d} \cdot \delta_{\vec{k}_{\|}, \vec{q}_{\|}},
$$

again assuming $\Delta \mu \ll \bar{\mu}$. These matrix elements vanish linearly with the incoming momentum at low energies, as expected for a tunneling amplitude. In order to avoid the related unlimited increase of the amplitudes for large momenta, we have introduced a cutoff $1 / \sqrt{1+(k \xi)^{2}}$ which leads to a saturation of the matrix elements at an energy scale still much smaller than the barrier height $V_{B}$. In fact for energies in this range, the transfer Hamiltonian fails, because the coupling between the two sides can no longer be treated perturbatively. Fortunately, for small chemical potential differences $\Delta \mu \ll \bar{\mu}$, only the low energy phonon like excitations with sound velocity $c=\sqrt{\mu / m}$ contribute to $J_{n}$ and $J_{2}$. Indeed, for small frequencies, the spectral functions are antisymmetric in $\omega$ and are equal up to a sign

$$
A(k, \omega) \simeq-B(k, \omega)=\frac{\pi \mu_{a}}{\hbar \omega} \delta\left(\omega-c_{a} k\right) \quad(\omega>0) .
$$

The sum over momenta is thus restricted to a regime where the cutoff is irrelevant. It plays a role only for the less important second order Josephson current $J_{1}$, which is small compared to the leading first order term, with a typical magnitude

$$
J_{1}(\Delta \mu=0) \approx I_{c} \cdot e^{-\kappa_{\bar{\mu}} d} / \kappa_{\bar{\mu}} \xi .
$$

Using (55), the c-nc contributions to $J_{n}$ and $J_{2}$ are readily evaluated in the limit $L \rightarrow \infty$, where the one particle energy spectrum becomes continuous. To lowest order in the chemical potential difference, both currents are linear in $\Delta \mu$, behaving like

$$
J_{n}(\Delta \mu \rightarrow 0)=J_{2}(\Delta \mu \rightarrow 0)=G_{n} \Delta \mu .
$$

The associated normal conductance per area for a symmetric situation is finite at zero temperature and given by

$$
G_{n} / A=\frac{2 \sqrt{2} n_{c}}{\hbar \kappa_{\bar{\mu}}^{2} \xi} \cdot e^{-2 \kappa_{\bar{\mu}} d} .
$$

Concerning the nc-nc terms, it is straightforward to see that their contribution to $G_{n}$ vanishes like $T^{4}$ and thus is negligible. The same applies to the nc-nc contribution to $J_{1}(\Delta \mu=0)$ which, as noted in section 3 , turns out to be smaller than the c-nc term (56) by a factor $\sqrt{n a^{3}}$ [29].

A remarkable feature of tunneling between Bose condensates is the presence of a normal conductance proportional to the condensate density. Contrary to the situation encountered in superconductors, the dissipation in a Bose Josephson junction thus remains finite even at $T=0$. It is obvious that this result relies on the continous excitation spectrum of the homogeneous system, and thus its relevance to BECs in traps might be questioned. I practice, though, for $\Delta \mu \gtrsim \hbar \omega$ and for the experimentally relevant temperatures where $k_{B} T \gg \hbar \omega$, the discreteness of the spectrum in the harmonic traps is irrelevant, and the continuum approximation thus well justified. We shall see, in the next section, that dissipative currents play an important role in the dynamics of coupled BECs, even if they are small. 


\section{DYNAMICS FOR REALISTIC PARAMETERS}

On the basis of our microscopic model developed in the previous sections, we now aim to provide realistic estimates for the necessary requirements and the expected dynamics in a Bose Josephson junction, which may be realized experimentally with present techniques. Assuming a contact area $A=20 \mu \mathrm{m}^{2}$ and a condensate extending about $10 \mu \mathrm{m}$ in the direction perpendicular to the barrier, we have roughly $\bar{N}_{a}=\bar{N}_{b}=10^{5}$ atoms on each side on average. With the known mass and scattering length the associated mean density $n=5 \times 10^{14} \mathrm{~cm}^{-3}$ then determines the average chemical potential $\bar{\mu}=h \times 8.2 \mathrm{kHz}$ for ${ }^{23} \mathrm{Na}$, which is the more favorable case for observing tunneling due its lighter mass compared with ${ }^{87} \mathrm{Rb}$. Since the tunneling amplitude decreases exponentially with barrier thickness $d$, it is desirable to have widths $d$ as small as possible. With barriers realized by a blue detuned laser, $d=1 \mu \mathrm{m}$ is difficult to reach, but still a realistic value. Using (49), the resulting critical current can now be determined for any given barrier height. With a large barrier $V_{B}=5 \bar{\mu}$, however, the resulting critical current is only $I_{c}=584 \mathrm{sec}^{-1}$, which is far too small to be observable. Indeed, the corresponding tiny Josephson coupling energy is not sufficient to establish phase coherence across the barrier against even the minute thermal fluctuations at the typical temperatures of gaseous BECs. In fact, these fluctuations give rise to a smearing $\left\langle\Delta \varphi^{2}\right\rangle=k_{B} T / E_{J}$ of the relative phase. A Josephson effect, which requires small phase fluctuations $\left\langle\Delta \varphi^{2}\right\rangle \ll 1$, is thus only possible if $I_{c} \gg k_{B} T / \hbar \simeq 10^{4} \mathrm{sec}^{-1}$ at $T=100 \mathrm{nK}$. For conventional superconductors, where $T$ is of the order of $1 \mathrm{~K}$, this condition expresses the well known fact that minimum critical currents are around $1 \mu \mathrm{A}$ 33.

In order to observe Josephson tunneling in gaseous BECs, one therefore needs barriers which are not much larger than the average chemical potential. For a quantitative estimate, it is important to consider the necessary magnitude of the oscillating currents, which are detectable as a clear signal of the Josephson effect against experimental noise. For a given difference $\Delta \mu$ in the chemical potentials, the amplitude of the oscillation in the number of particles is $E_{J} / \Delta \mu$. With current experimental resolution, this amplitude should be at least around five percent of the total particle number to be detectable. Using typical values $\Delta \mu=0.02 \bar{\mu}$, this requires Josephson coupling energies $E_{J} \gtrsim 10^{-3}\left(N_{a}+N_{b}\right) \bar{\mu}$ (note that this is still consistent with the requirement $E_{J} \ll\left(N_{a}+N_{b}\right) \bar{\mu}$ of a tunneling Hamiltonian description). With the numbers above, this translates into critical currents $I_{c} \gtrsim 10^{7} \mathrm{sec}^{-1}$, putting a much stronger limit than that of negligible thermal fluctuations. To realize a Josephson contact in the required range, it is necessary to choose a rather small barrier height $V_{B}=1.25 \bar{\mu}$. The critical current in a condensate with the above pa- rameters is then around $4 \cdot 10^{6} \mathrm{sec}^{-1}$. For ${ }^{23} \mathrm{Na}$, the observation of a Josephson effect is therefore close to the limit of detectability, and may be possible in an optimized setup. By contrast, for ${ }^{87} \mathrm{Rb}$, the achievable critical currents even for barriers as low as $1.25 \bar{\mu}$ are an order of magnitude smaller than those in ${ }^{23} \mathrm{Na}$. Thus it appears unlikely, that coherent transfer of atoms across a tunnel barrer may be realized in this case with presently available condensates.

For a quantitative analysis, it is necessary to consider the time evolution of the relative particle number $\nu=\left(N_{a}-N_{b}\right) / 2$ including 'charging' effects and dissipation. As noted in section 5 , the total difference $\Delta \mu$ in the chemical potential is a sum of a possible external contribution $\Delta \mu_{0}$ (induced e.g. by a difference in the gravitational potentials as realized in [15]) plus an internal 'charging' term $U \nu$ associated with a difference in the densities due to particle transfer. The generally valid Josephson relation (23) thus reads

$$
\hbar \dot{\varphi}=-\Delta \mu_{0}-U \nu
$$

For a constant $\Delta \mu_{0}$, a further time derivative then leads to the semiclassical equation of motion

$$
\begin{aligned}
& \frac{\hbar}{U} \ddot{\varphi}(t)+G_{n} \hbar \dot{\varphi}(t)(1+\cos 2 \varphi(t)) \\
& \quad+I_{c} \sin \varphi(t)+J_{1}(-\hbar \dot{\varphi}(t)) \sin 2 \varphi(t)=0
\end{aligned}
$$

provided $-\dot{\nu}$ is replaced by the total current obtained for a given instantaneous $\Delta \mu=-\hbar \dot{\varphi}$. Obviously this is just Eq.(42) without the fluctuating forces, determining the dynamcis of the averaged relative phase and the associated mean current. Eq.(60) is the analog of the well known resistively shunted junction model for superconducting Josephson junctions [33], and is valid for small phase fluctuations $\left\langle\Delta \varphi^{2}\right\rangle \ll 1$. Apart from the condition $E_{J} \gg k_{B} T$ mentioned above, this also requires that the Josephson coupling is much larger than the charging energy $U$. Now, in a symmetric situation with $\bar{N}_{a}=\bar{N}_{b}=\bar{N}$ and for the parameters choosen above, $U=2 \bar{\mu} / \bar{N}$ is only of order $h \times 0.16 \mathrm{~Hz}$ compared with $E_{J}=h \times 0.6 \mathrm{MHz}$. Nevertheless, charging effects are far from negligible, since for small values of $\Delta \mu_{0}$ they are the dominant contribution in (59) driving the phase dynamics. To give a quantitative example, we have numerically integrated the averaged, semiclassical time evolution of $\varphi$ and $\nu$ for the above parameters, neglecting second order currents. As is shown in Fig. 2, the normalized particle number difference $z=\nu / \bar{N}$ for a constant offset potential $\Delta \mu_{0}=0.03 \bar{\mu}$ and initial condition $\nu(0)=0$ exhibits appreciable deviations from a pure sinusoidal oscillation predicted in the absence of 'charging' effects. Obviously both the amplitude and the frequency of the Josephson oscillations are modified, although only by a factor of order of unity. The typical time scale for the oscillations is msec, which is well within the reach of experimental observation. 


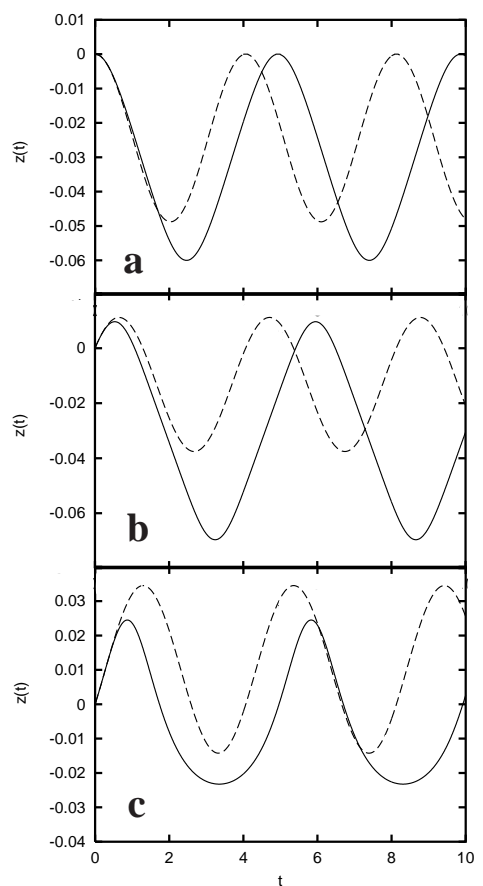

FIG. 2. Nonlinearity of the Josephson oscillations due to charging effects. For the parameters discussed in the text, the normalized population difference is shown for initial relative phases $\varphi=0,1,2$ (from top) both with (solid line) or without charging effects (dashed line). The time is measured in msec.

Regarding the higher order and dissipative currents, which have been neglected in Fig. 2, it turns out that for the low barriers choosen here, the amplitude of the second order Josephson current is not much smaller than the leading term, with a typical value $J_{1}(\Delta \mu=0) \simeq 0.1 I_{c}$. Now although this contribution oscillates at twice the frequency of the first order term, an experimental detection is probably out of reach at present, given the narrow margin for seeing even the dominant effect, as discussed above. The dissipative normal and interference currents, however, significantly change the dynamics of the coupled condensates and thus should be indirectly observable even if the individual contributions are tiny. Indeed from Eq. (60) it is evident, that these currents lead to a damping of the phase dynamics with a typical time scale of order $\tau=1 / G_{n} U$. For the parameters choosen above, the normal conductance is $G_{n}=250 / h$, giving a relaxation time $\tau=0.025 \mathrm{~s}$. The amplitude of the Josephson oscillations will therefore decay to $1 / e$ of their inititial value after typically 25 periods. As pointed out by $\mathrm{Ru}-$ ostekoski and Walls [34], dissipative currents also eliminate the macroscopic quantum self trapping predicted by Smerzi et.al. [16,35] for systems with a large initial population imbalance and small Josephson coupling. For a quantitative estimate, we consider the influence of an ohmic normal current $J_{n}=G_{n} \Delta \mu$ in a simplified equation of motion for the normalized particle number difference $z$. With $\tilde{t}=2 t_{c c} t / \hbar$ as a dimensionless time and for a symmetric situation with $\Delta \mu_{0}=0$ they read

$$
\frac{d z}{d \tilde{t}}=-\sqrt{1-z^{2}} \sin \varphi-2 \hbar G_{n} \bar{\mu} / E_{J} \cdot z
$$

and

$$
\frac{d \varphi}{d \tilde{t}}=-\Lambda z-\frac{z}{\sqrt{1-z^{2}}} \cos \varphi .
$$

Here $\Lambda=U \bar{N} / 2 t_{c c}=\bar{\mu} / t_{c c}$ is essentially the ratio between the condensate ground state energy $E_{0} \approx \bar{\mu} \bar{N}$ and the Josephson coupling energy $E_{J}=2 t_{c c} \bar{N}$. The $\cos \varphi$ contribution in (62) arises from the $z$-dependence of the critical current $I_{c}(z)=I_{c} \cdot \sqrt{1-z^{2}}$, relevant for large asymmetries $z=O(1)$, while the second order Josephson currents discussed above are neglected for simplicity [36]. The phenomenological equations $(61,62)$ lead to a self trapping of the condensate, provided $\Lambda$ is larger than a critical value which depends on the initial asymmetry $z(0)$. In the absence of dissipation, the asymmetry will be maintained in time, leading to a behaviour as shown in Fig. 3 (dotted line), with $z(t)$ oscillating near its initial value $z(0)=0.6$ (as in ref. [16] we choose $\Lambda=11$ and $\varphi(0)=0)$.

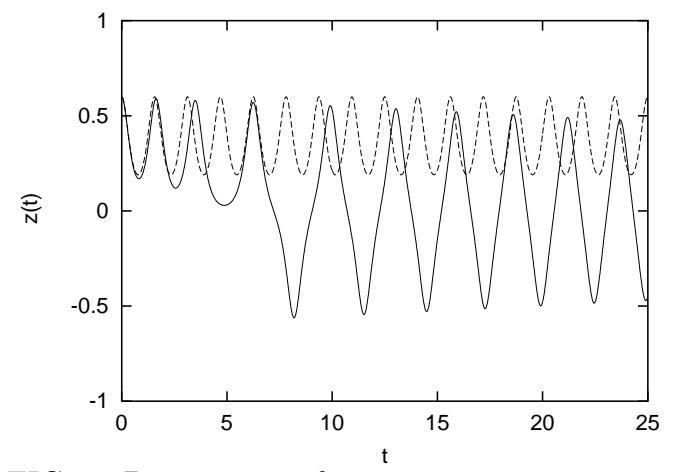

FIG. 3. Destruction of macroscopic quantum self trapping through dissipative currents. For comparison, the dynamics obtained without dissipation is also shown (dashed line). Time is measured in units of $\hbar / 2 t_{c c}$ and thus with a typical value $t_{c c}=h \times 20 \mathrm{~Hz}, t=25$ corresponds to about $0.1 \mathrm{sec}$

It is obvious that normal currents, which lead to an equilibration of the chemical potentials $\Delta \mu=\mu_{a}-\mu_{b} \rightarrow 0$ for long times, will eventually destroy a self trapped state. Observation of macroscopic quantum self trapping is therefore possible only if the time scale $1 / G_{n} U$ for equilibration is much larger than $\hbar / 2 t_{c c}$, which is the typical scale for the dynamics in the trapped state. This requires that the conductance obeys $\hbar G_{n} \ll E_{J} / 2 \bar{\mu}$, i.e. the coefficient in front of the dissipative term in (61) should be small compared to one. Now from our microscopic results (49) and (58), we find that

$$
2 \hbar G_{n} \bar{\mu} / E_{J}=\frac{2 \sqrt{2}}{f\left(V_{B} / \bar{\mu}\right) \kappa_{\bar{\mu}} \xi} \cdot e^{-\kappa_{\bar{\mu}} d} .
$$

For high barriers, with a corresponding tiny Josephson coupling energy, this ratio can certainly be made small 
enough, such that dissipation has essentially no influence on the dynamics. As pointed out above, however, realistic systems allowing to observe Josephson tunneling require small barriers. For those, the inequality is strongly violated and in fact, for the parameters choosen above, the ratio in (63) is close to one. For a quantitative example, which allows a comparison with the results obtained in ref. 16], we assume a condensate with much lower density $n \approx 1.3 \cdot 10^{13} \mathrm{~cm}^{-3}$ and a comparatively large value $t_{c c}=h \times 20 \mathrm{~Hz}$, giving $\Lambda=11$ [37]. With reasonable parameters $V_{B} \approx 2 \bar{\mu}, \kappa_{\bar{\mu}} d=4.2$ and $\kappa_{\bar{\mu}} \xi=3$, the ratio in (63) has a rather small value 0.0275. The resulting time evolution of $z(t)$ including dissipation, is shown as the solid line in Fig. 3. Evidently self trapping is destroyed rather quickly even in this case and in fact, the situation does not change qualitatively for other parameters which seem accessible. From our microscopic results, it thus appears unlikely that macroscopic quantum self trapping can actually be observed in condensates which are realizable at present.

Finally we want to discuss the problem, to which extent the tunneling Hamiltonian is still applicable in a regime, where the barrier $V_{B}$ is only slightly larger than the chemical potential, as was assumed above. Now at least on the mean-field-level, the quality of the transfer Hamiltonian model may be tested by comparing its predictions with those obtained from a numerical integration of the time dependent GPE in the geometry of Fig. 11. Due to the periodic boundary conditions in the directions parallel to the potential barrier, the problem is effectively one-dimensional. The time evolution of the condensate wavefunction can thus be easily determined using a split-operator Fourier technique. In Fig. 1, the normalized number of particles which have tunneled through the barrier is shown for a case with $d=2 \xi \approx 0.3 \mu \mathrm{m}$ and a comparatively large value $\Delta \mu_{0}=0.2 \bar{\mu}$. Time is measured in units $\hbar / \bar{\mu}$, corresponding to about $0.6 \mathrm{msec}$ at $t=30$ for our values above. The height of the potential barrier is lowered continuously from an initial value $10 \bar{\mu}$

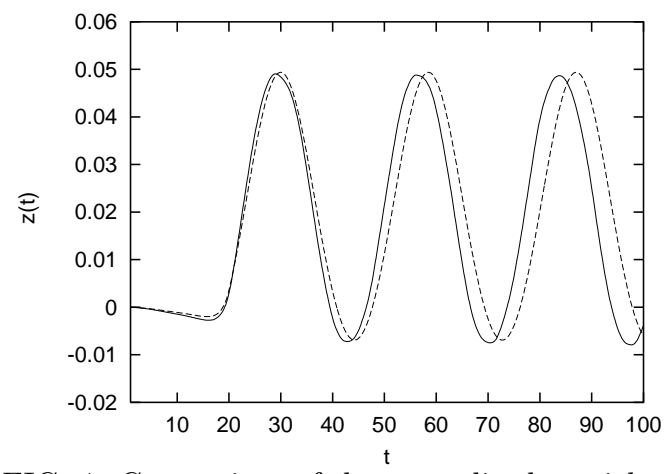

FIG. 4. Comparison of the normalized particle number difference $z(t)$ obtained by a numerical integration of the GPE (solid line) and from the semiclassical dynamics based on the tunneling Hamiltonian (dashed line) for a high and narrow potential barrier with $d=2 \xi$ and $V_{B}=3 \bar{\mu}$ at $t=10$ to $V_{B}=3 \bar{\mu}$ at $t=20$. Charging effects are contained intrinsically in the GPE or via the $U \nu$ contribution to the chemical potential difference; the initial conditions are $\nu(0)=\varphi(0)=0$. Evidently, the result obtained within the transfer Hamiltonian model is in excellent agreement with that from a numerical solution of the GPE, in the limit of a high and narrow barrier. Note that there are no adjustable parameters here, with all quantities being determined by the microscopic parameters of the Bose system. Remarkably, the transfer Hamiltonian still provides a reasonable approximation even for the case of relatively wide and low potential barriers, which are relevant experimentally. For example, in Fig. 5, the function $z(t)$ is plotted for an identical situation as above, however with $d=5 \xi \approx 0.8 \mu \mathrm{m}, V_{B}=1.4 \bar{\mu}$ and $\Delta \mu_{0}=0.1 \bar{\mu}$ Although the semiclassical dynamics based on the transfer Hamiltonian does not reproduce the higher harmonics present in the solution of the GPE, which are essentially a result of the nonadiabatic lowering of the barrier, it still provides a reasonable approximation even in this rather extreme case of a barrier which is only slightly larger than $\bar{\mu}$.

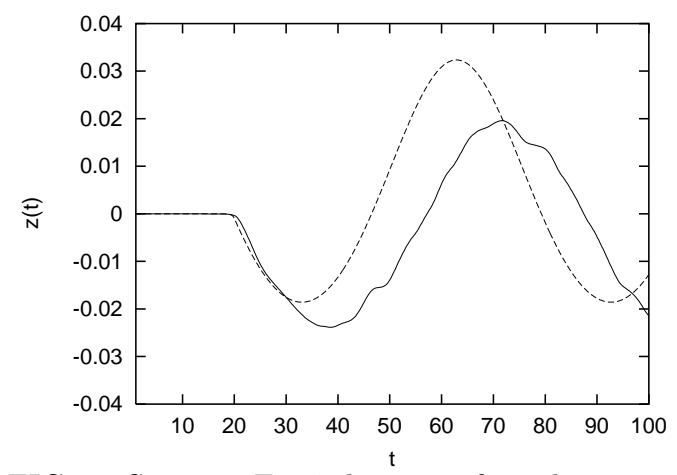

FIG. 5. Same as Fig.4, but now for a barrier with $d=5 \xi$ and $V_{B}=1.4 \bar{\mu}$

\section{CONCLUSIONS AND OUTLOOK}

In summary, we have developed a microscopic theory of Josephson tunneling in weakly interacting BECs. It is essentially the analog within Bogoliubov theory of the standard work by Ambegaokar et.al. 24, 31 on the tunneling Hamiltonian description of the Josephson effect between BCS superconductors. Apart from the fact that the Bogoliubov theory only applies far below the condensation temperature $T_{c}$, while BCS is valid right up to $T_{c}$, there are a number of further crucial differences. Most importantly, the Josephson effect in BECs arises already in first order in the tunneling amplitude. As a result, the Josephson current dominates any other contributions in the limit of weakly coupled condensates, to which our discussion has been restricted. Nevertheless, the second order dissipative currents have a strong influence on the dynamics of coupled condensates, because they remain finite even at zero temperature due to the 
absence of a gap. Remarkably, the dominant contribution to the normal current, which leads to a damping of the Josephson oscillations, arises from c-nc tunneling and is thus proportional to the condensate density. The explicit calculation of the total current has been performed here in a very simplified model. It has the advantage, however, in providing analytical and physically transparent results, allowing to determine the currents in a concrete experimental realization from the knowlegde of the bulk condensate properties, the barrier height and the effective contact area. The fact that tunneling in a Bose system is possible at all energies, made it necessary to specify the associated matrix elements in much more detail than in superconductors. There, only the Fermi energy is relevant, and thus the matrix elements can be replaced by a constant which is fixed by the normal state conductance. The effect of 'charging' for condensates with a constant total particle number has been shown to be quantitatively important in realistic situations, even though the relevant energy $U$ is much smaller than the Josephson coupling energy $E_{J}$.

Regarding the prospects for an experimental observation of the Josephson effect in condensates of dilute atomic gases, our estimates show that this requires barriers which are only slightly higher than the chemical potential. With presently available condensates, the observation appears to be possible with ${ }^{23} \mathrm{Na}$, not, however, with heavier atoms. One of the problems, for instance, which may suppress the small oscillating Josephson currents, are random fluctuations in the barrier height due to fluctuations in the laser intensity, a complication which has not been considered so far.

On the theoretical side, quantitative calculations for realistic geometries of coupled traps (in particular beyond the Gross-Pitaevski level) would be useful and also a careful consideration of finite temperature effects. For example, the currents due to particles in the thermal cloud have been estimated in [17], however more work needs to be done in this direction. Finally, since Josephson oscillation frequencies are typically of the same order than those of collective modes [5] of the individual condensates, it is important to investigate a possible coupling between the intra- and inter-well dynamics.

We gratefully acknowledge helpful discussions with $\mathrm{T}$. Esslinger and I. Bloch on the experimental aspects of our work.

[1] M.H. Anderson et al., Science 269, 198 (1995)

[2] K.B. Davis et al., Phys. Rev. Lett 75, 3969 (1995)

[3] C.C. Bradley, C.A. Sacket, J.T. Tollett, and R.G. Hulet, Phys. Rev. Lett. 75, 1687 (1995)

[4] G.Baym and C.J. Pethick, Phys. Rev. Lett. 76, 6 (1996)

[5] F. Dalfovo, S. Giorgini, L.P. Pitaevskii, and S. Stringari,
Rev. Mod. Phys. 71, 463 (1999)

[6] M.R. Andrews et al., Phys. Rev. Lett. 79, 553 (1997)

[7] M.R. Matthews et al., Phys. Rev. Lett. 83, 2498 (1999)

[8] K.W. Madison, F. Chevy, W. Wohlleben and J. Dalibard, Phys. Rev. Lett. 84, 806 (2000)

[9] C. Raman et al., Phys. Rev. Lett. 83, 2502 (1999)

[10] B. D. Josephson, Phys. Lett. 1, 251 (1962)

[11] B.D. Josephson in R.D. Parks(ed.) Superconductivity, Vol. 1, New York, 1969

[12] P.W. Anderson, Rev. Mod. Phys. 38, 298 (1966)

[13] O. Avenel and E. Varoquaux, Phys. Rev. Lett. 55, 2704 (1985)

[14] D. S. Hall, M.R. Matthews, C.E. Wieman, and E.A. Cornell, Phys. Rev. Lett 81, 1543 (1998)

[15] B.P. Anderson and M.A. Kasevich, Science 282, 1686 (1998)

[16] A. Smerzi, S. Fantoni, S. Giovanazzi, and S.R. Shenoy, Phys. Rev. Lett. 79, 4950 (1997)

[17] I. Zapata, F. Sols, and A.J. Leggett, Phys. Rev. A 57, R28 (1998)

[18] P. Villain and M. Lewenstein, Phys. Rev. A 59, 2250 (1999)

[19] A brief account of our main results was given previously, see F. Meier and W. Zwerger, cond-mat/9904147

[20] C.B. Duke in F. Seitz, D. Turnbull, and H. Ehrenreich (ed.) Solid State Physics, Suppl. 10, New York, 1969

[21] R.E. Prange, Phys. Rev. 131, 1083 (1963)

[22] R.A. Ferrell and R.E. Prange, Phys. Rev. Lett. 10, 479 (1963)

[23] F. Bloch, Phys. Rev. B2, 109 (1970)

[24] V. Ambegaokar and A. Baratoff, Phys. Rev. Lett. 10, 486 (1963) and Phys. Rev. Lett. 11, 104 (1963) (E)

[25] S. Giorgini, L.P.Pitaevski and S. Stringari, J. Low Temp. Phys. 109, 309 (1997)

[26] R.A. Ferrell, Phys. Rev. B38, 4984 (1988)

[27] A. L. Fetter and J. D. Walecka, Quantum Theory of Many- Particle Systems, New York 1971

[28] P. Nozieres, Liquides et Solides quantiques, Lecture Notes (1983), unpublished

[29] F. Meier, Josephson-Effekte in gekoppelten Bose- Kondensaten, Diploma thesis, Ludwig-Maximilians- Universität München, 1999, unpublished

[30] W. Zwerger, Solid State Comm. 45, 841 (1983)

[31] V. Ambegaokar, U. Eckern, and G. Schön, Phys. Rev. Lett. 48, 1745 (1982); U. Eckern, G. Schön, and V. Ambegaokar, Phys. Rev. B 30, 6419 (1984)

[32] J.W. Negele and H. Orland, Quantum Many-Particle Systems, Addison-Wesley, 1988

[33] M. Tinkham, Introduction to Superconductivity, McGraw-Hill 1996

[34] J. Ruostekoski and D. Walls, Phys. Rev. A58, R50 (1998)

[35] The phenomenon was in fact first predicted by M. Kleber and H. Schmidt, Z. Physik. 245, 68 (1971) for Josephson tunneling between colliding superfluid nuclei.

[36] As mentioned in section 6, for $z=O(1)$ the tunneling amplitude $t_{c c}$ is itself $z$-dependent, an effect which is not taken into account here. Note also, that our $\varphi$ has the opposite sign as the one in ref. [16].

[37] The value $\Lambda=11$ is rather small, for our parameters above $\Lambda$ is in fact around 2500 . 